\title{
Ulcerative colitis may be a risk factor for sensorineural hearing loss
}

\author{
๑DAhmet Yozgat ${ }^{1}$, @Müjgan Gürler ${ }^{2}$ \\ ${ }^{1}$ Ufuk University, Faculty of Medicine, Department of Gastroenterology, Ankara, Turkey \\ ${ }^{2}$ Abant İzzet Baysal University, Faculty of Medicine, Department of Internal Medicine, Bolu, Turkey
}

Cite this article as: Yozgat A, Gürler M. Ulcerative colitis may be a risk factor for sensorineural hearing loss. J Health Sci Med 2021; 4(3): 267-271.

\begin{abstract}
Introduction: Inner ear involvement of inflammatory bowel disease (IBD), which causes sensorineural hearing loss (SNHL) is acute and bilateral and arises in a short period of weeks to months in the active period of the disease. We aimed to determine the frequency of SNHL in patients with UC and CD and to evaluate the relationship between audiological features and clinical findings in IBD.

Material and Method: The present study included 53 IBD patients and 20 healthy control patients who were followed up in the gastroenterology outpatient clinic of Abant Izzet Baysal University Hospital between January and May 2020 and accepted to participate in the study. Tympanometry, otoscopy, and audiometry examinations were performed.

Results: There was no significant difference in terms of gender and age between the IBD and control groups. While there was no significant difference in air and bone conduction in both ears in patients with $\mathrm{CD}$, there was a significant difference between both conductions in UC (p: 0.0001 in the left ear, p: 0.004 in the right ear). SNHL was detected in 45.2\% (n:14) of UC patients and $13.6 \%(n: 3)$ of CD patients in audiometry. Three of our UC patients had moderate, one had moderately severe, and one had profound hearing loss.

Conclusion: SNHL has been detected in a significant number of UC patients. Also, the hearing functions deteriorate significantly as the age of the patients and the duration of the disease increases. UC patients with a long-term disease or older patients should be evaluated for SNHL.
\end{abstract}

Keywords: Sensorineural hearing loss, inflammatory bowel disease, ulcerative colitis, Crohn's disease, audiometry

\section{INTRODUCTION}

Inflammatory bowel disease (IBD) is a chronic, idiopathic, relapsing, inflammatory disease of the gastrointestinal system, which includes two different diseases, namely ulcerative colitis (UC), and Crohn's disease (CD). Although IBD mainly affects the gastrointestinal system, it causes several extraintestinal symptoms as in all chronic inflammatory diseases (1). Extraintestinal symptoms can be divided into extraintestinal complications and extraintestinal manifestations (EIM). EIM are extraintestinal findings that develop due to a process similar to gut inflammation. The most common EIM are; rheumatologic (ankylosing spondylitis and peripheral arthropathies), hepatobiliary (primary sclerosing cholangitis), ophthalmologic (uveitis, episcleritis), and cutaneous (stomatitis, erythema nodosum, pyoderma gangrenosum, Sweet's Syndrome) (2). Extraintestinal complications, on the other hand, are findings such as anemia, nephrolithiasis, cholelithiasis, and osteoporosis due to the direct effect of intestinal inflammation (3).

Ear involvement in IBD is usually in the form of inner ear involvement, rarely, external ear involvement can be seen (4). Hearing loss in IBD patients was first described in 1982, in a UC patient with bilateral sensorineural hearing loss (SNHL) and vestibular dysfunction (5). Inner ear involvement, which causes sensorineural hearing loss, in particular, is associated with autoimmune inner ear disease (AIED) (6). SNHL associated with IBD often affects UC patients. Hearing loss is acute and bilateral and occurs in a short period of weeks to months in the active period of the disease (4). In a few studies, significant levels of SNHL have been reported, especially in UC patients, but in some studies, these rates were found to be lower (7-10). Therefore we aimed to determine the frequency 
of SNHL in patients with UC and CD and to evaluate the relationship between audiological features and clinical findings in IBD.

\section{MATERIAL AND METHOD}

\section{Ethical Issue}

The study was approved by the Bolu Abant İzzet Baysal University Clinical Research Ethics Committee (Date: 07.11.2019, Decision No: 2019/111). All procedures were carried out in accordance with the ethical rules and the Declaration of Helsinki.

\section{Patients}

The present study included 53 IBD patients $(22 \mathrm{CD}$ and 31 UC patients) and 20 healthy control cases who were followed up in the gastroenterology outpatient clinic of Abant Izzet Baysal University Hospital between January and May 2020 and accepted to participate in the study. Healthy volunteers and patients underwent a complete otorhinolaryngeal and audiometric evaluation, and informed consent was obtained from all participants. Patients with a known ear-related disease, chronic systemic disease, head trauma, exposure to loud noise, and use of the ototoxic drugs in the last 2 years were excluded from the study. Patients' personal and demographic information, medical history, comorbidities, medications they used, smoking-alcohol use, duration of illness, age of diagnosis, disease activity, routine laboratory data were recorded from the medical records of the patients. To evaluate disease activity; the Mayo score was used in UC patients and Harvey Bradshaw activity index on CD $(11,12)$. As the control group, patients who do not have a known disease and routine drug usage were selected by matching IBD patients according to age and gender.

\section{Audiometry}

Tympanometry, otoscopy, and pure tone audiometry (PTA) (air conduction at; 0.25, 0.5, 1, 2, 4, and $8 \mathrm{kHz}$ and bone conduction at; $0.5,1,2,4 \mathrm{kHz}$ ) were performed on the healthy controls and IBD patients. In the audiometric examination, air and bone conduction threshold values from both ears were determined, and the mean hearing threshold level was accepted as hearing level. Besides, speech discrimination percentage (\%), speech perception threshold values (decibels $(\mathrm{dB})$ ), and the most comfortable loudness threshold values $(\mathrm{dB})$ were also determined. The normal hearing threshold value in the audiogram is accepted as 15 decibels or less. The hearing threshold value between 41-55 decibels is accepted as moderate hearing loss, 56-70 moderately severe, 71-90 severe, and more than 90 is accepted as profound hearing loss (13). In our study, a hearing threshold level above $20 \mathrm{~dB}$ was accepted as SNHL. Those with a conduction difference between air and bone greater than 10 decibels were considered as conductive hearing loss and excluded from the study.

\section{Statistical Analysis}

One-way Anova and Student-t-test (Man-Whitney U and Kruskal Wallis tests were used for nonparametric variables) were used to evaluate continuous variables between groups, and the chi-square (X2) test was used for categorical variables. Correlation between data was evaluated by Spearman rank correlation test or Pearson test according to whether the data had normal distribution or not. SPSS 22.0 program (Armonk, NY: IBM Corp.) was used for statistical analysis. A significant (p) value was accepted as $<0.05$.

\section{RESULTS}

A total of 53 patients and 20 healthy volunteers participated in the study. There was no significant difference in terms of gender and age (mean age of UC: $49.4 \pm 14.1$; CD: $49.1 \pm 12.5$; controls: $48.5 \pm 11.5$ p:0.95) in the patients and control groups. $72.7 \%$ of UC patients, $71 \%$ of $\mathrm{CD}$ and $70 \%$ of controls were male (p:0.94). The general clinical characteristics of the patients are summarized in Table 1.

\begin{tabular}{|c|c|c|c|}
\hline & $\begin{array}{l}\text { Crohn's } \\
\text { disease } \\
(\mathrm{n}: 22)\end{array}$ & $\begin{array}{l}\text { Ulcerative } \\
\text { colitis } \\
(\mathrm{n}: 31)\end{array}$ & $\mathbf{p}$ \\
\hline Gender. n (\%) & & & 0.89 \\
\hline Female & $6(27.3)$ & $9(29)$ & \\
\hline Male & $16(72.7)$ & $22(71)$ & \\
\hline Age. (year, mean \pm SD) & $49.1 \pm 12.5$ & $49.4 \pm 14.1$ & 0.93 \\
\hline Activity, n (\%) ${ }^{1}$ & & & 0.78 \\
\hline Active & $5(22.7)$ & $8(25.8)$ & \\
\hline Remission & $17(77.3)$ & $23(74.2)$ & \\
\hline Duration, $(\text { month mean } \pm \mathrm{SD})^{2}$ & $73.3 \pm 48.8$ & $89.3 \pm 102.7$ & 0.45 \\
\hline Behavior, CD n (\%) & & & N/A \\
\hline Nonstricturing-nonpenetrating & $20(90.9)$ & - & \\
\hline Stricturing & $1(4.5)$ & - & \\
\hline Penetrating & $1(4.5)$ & - & \\
\hline Location, CD n (\%) & & & N/A \\
\hline Ileal & $3(13.6)$ & - & \\
\hline Ileocolonic & $13(59.1)$ & - & \\
\hline Colonic & $3(13.6)$ & - & \\
\hline Perianal & $3(13.6)$ & - & \\
\hline Location, UC n (\%) & & & N/A \\
\hline Proctitis & - & $4(12.9)$ & \\
\hline Left-sided colitis & - & $11(35.5)$ & \\
\hline Extensive colitis & - & $16(51.6)$ & \\
\hline \multicolumn{4}{|l|}{ Therapy, n (\%) ${ }^{1}$} \\
\hline Mesalazine & $17(77.3)$ & $30(96.8)$ & 0.07 \\
\hline Methylprednisolone & $5(22.7)$ & $3(9.7)$ & 0.25 \\
\hline Azathioprine & $9(40.9)$ & $1(3.2)$ & 0.001 \\
\hline Anti-TNFa & $5(22.7)$ & $4(12.9)$ & 0.46 \\
\hline
\end{tabular}


While there was no significant difference in air and bone conduction in both ears in $\mathrm{CD}$, there was a significant difference in both conductions in UC (p: 0.0001 in the left ear, p: 0.004 in the right ear). Hearing threshold values in UC patients were higher than that of the control group. Both air and bone hearing threshold values in the left ear were significantly higher in UC than CD (p:0.008, $\mathrm{p}: 0.007$, respectively). There was no significant difference between values in the right ear. SNHL was detected in 45.2\% (n:14) of UC patients and $13.6 \%$ (n:3) of CD patients in PTA. None of the volunteers in the control group had SNHL between $0.5-4 \mathrm{kHz}$, four had SNHL between 4-8 $\mathrm{kHz}$. Three of our UC patients had moderate, one had moderately severe, and one had profound hearing loss. The audiological data are summarized in Table 2. SNHL was similar between groups in terms of behavior and location pattern in both diseases.

\begin{tabular}{|c|c|c|c|c|c|}
\hline & Control & CD & $\mathbf{p}^{1}$ & UC & $\mathbf{p}^{2}$ \\
\hline \multicolumn{6}{|c|}{ Mean hearing level $(\mathrm{dB})$ at $0.5-4 \mathrm{kHz}$ frequencies } \\
\hline \multicolumn{6}{|c|}{ Air $($ mean \pm SD) } \\
\hline Right & $12.6 \pm 4.9$ & $20.7 \pm 20.3$ & 0.08 & $26.0 \pm 17.1$ & 0.0001 \\
\hline Left & $12.3 \pm 5.4$ & $13.0 \pm 8.4$ & 0.74 & $25.1 \pm 22.2$ & 0.004 \\
\hline \multicolumn{6}{|c|}{ Bone $($ mean \pm SD $)$} \\
\hline Right & $8.3 \pm 5.2$ & $14.4 \pm 15.1$ & 0.09 & $20.2 \pm 15.4$ & 0.0001 \\
\hline Left & $8.8 \pm 5.7$ & $9.6 \pm 6.8$ & 0.69 & $18.5 \pm 15.7$ & 0.004 \\
\hline \multicolumn{6}{|c|}{ Speech perception threshold values $(\mathrm{dB})($ mean $\pm \mathrm{SD})$} \\
\hline Right & $9.0 \pm 4.8$ & $13.9 \pm 16.5$ & 0.21 & $17.7 \pm 13.7$ & 0.002 \\
\hline Left & $10.3 \pm 5.9$ & $11.4 \pm 7.1$ & 0.59 & $18.2 \pm 16.2$ & 0.017 \\
\hline \multicolumn{6}{|c|}{ Most comfortable loudness threshold values $(\mathrm{dB})($ mean $\pm \mathrm{SD})$} \\
\hline Right & $49.0 \pm 4.8$ & $52.7 \pm 12.0$ & 0.20 & $57.3 \pm 12.0$ & 0.001 \\
\hline Left & $50.3 \pm 6.0$ & $51.4 \pm 7.1$ & 0.59 & $57.1 \pm 12.0$ & 0.01 \\
\hline \multicolumn{6}{|c|}{ Hearing Loss } \\
\hline & & & $\mathrm{p}^{3}$ & & $\mathbf{p}^{4}$ \\
\hline \multicolumn{6}{|c|}{ SNHL $\mathbf{n}(\%)$} \\
\hline Right & $0(0)$ & $3(13.6)$ & 0.11 & $14(45.2)$ & 0.0001 \\
\hline Left & $0(0)$ & $4(18.2)$ & 0.11 & $11(35.5)$ & 0.001 \\
\hline
\end{tabular}

In UC, in air and bone conduction in the right ear, hearing is at a significantly higher volume in all frequencies except $0.25 \mathrm{kHz}(\mathrm{p}<0.01)$. On the left, hearing is at a higher volume in the middle and high frequencies $(\mathrm{p}<0.01)$ (Figure 1).

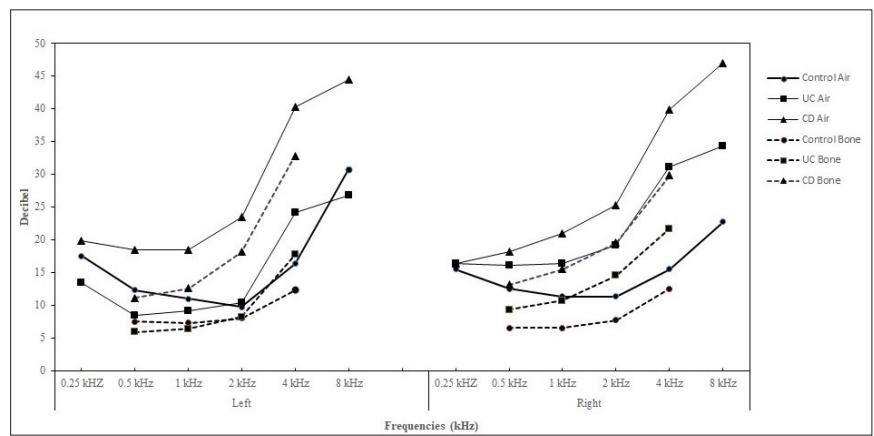

Figure 1. Comparison of hearing levels between diseases and control group (Left \& Right Ear).
When the correlation between the mean hearing levels at $0.5-4 \mathrm{kHz}$ frequencies and age was evaluated in UC patients, it was observed that the hearing functions decreased significantly as the age of the patients increased (p: 0.001). As the duration of the illness increases, there is a significant decrease in hearing functions only in air conduction in the left ear (p:0.02) (Figure 2). Disease activity had no effect on hearing level in patients with ulcerative colitis (p:0.94).

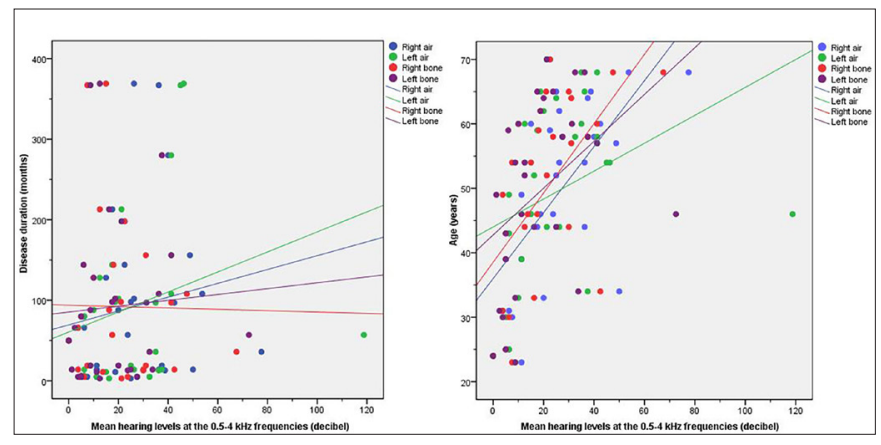

Figure 2. Correlation of mean hearing levels at $0.5-4 \mathrm{kHz}$ with age and duration of illness in patients with ulcerative colitis

\section{DISCUSSION}

SNHL is the most common cause of hearing loss, but SNHL of autoimmune origin is a rare cause (nearly $1.5 \%$ of all SNHL) (14). AEID was first described in 1979 (15) and has since been associated with many autoimmune diseases including Wegener's granulomatosis, Sjogren's syndrome, Behcet's disease, rheumatoid arthritis, and systemic lupus erythematosus (16). In various autoimmune diseases, it has been determined that SNHL is seen especially at high frequencies $(17,18)$.

The etiology of IBD has not been fully elucidated, and these patients have a non-infectious inflammation in the intestinal mucosa due to inappropriate immune response to the intestinal flora (19). Luminal antigens targeting immune-responsive organs are thought to be effective in the pathogenesis of EIM in IBD patients and the inner ear is considered one of them (10). SNHL due to inner ear involvement is an immune-mediated (AIED) disease and is associated with disease activity (4). Immunemediated damage of inner ear structures begins with the recognition of self-antigens by cochlear immune cells. Then vascular permeability increases and chemotaxis of activated cytotoxic $\mathrm{T}$ lymphocytes is facilitated by inflammatory mediators. As a result, a severe Th1 response and tissue damage occurs (20). SNHL in IBD patients can be in acute or silent form, or there may be periods of remission and exacerbations (9).

In the present study, we showed that SNHL increased significantly in UC patients at frequencies of $0.5-4 \mathrm{kHz}$ compared to control. However, in these patients, SNHL 
was at the subclinical level and none of the patients had complaints about hearing during the study period. While SNHL was detected in $45.2 \%$ of UC patients, this rate was $13.6 \%$ in $\mathrm{CD}$ and did not reach a statistically significant level. Although none of the volunteers in the control group had SNHL between $0.5-4 \mathrm{kHz}$, four had SNHL between 4-8 kHz. While many EIMs are observed in patients with UC, in recent years, reports of inner ear involvement and related SNHL have been increasing, and there are few and small-scale studies. In a study conducted by Akbayir et al., no significant difference was found between the control group and the patient group in terms of bilateral SNHL presence, while the mean hearing threshold values were higher in the patient group at high frequencies $(2-4-8 \mathrm{kHz})$. It was stated that the patients were asymptomatic and it was not known whether they could become symptomatic, and follow-up of the patients was recommended (7). In contrast, it was determined that only $2 \%$ of 57 UC patients developed SNHL in the 10-year period in a retrospective study performed by Casella et al (9). This finding may indicate that UC patients are less likely to develop symptomatic SNHL in 10 years. However, the results do not seem reliable enough because of the insufficient explanation of the methods and their retrospective design.

In a prospective study by Wengrower et al., $38 \%$ of all patients had SNHL, while this rate increased to $62 \%$ over the age of 40. It has been shown that patients with other EIMs have higher rates of SNHL, and it has been stated that SNHL can be considered as an EIM. It was determined that SNHL increased especially in patients over 40 years old and early evaluation of these high-risk patients was recommended (10). This is compatible with our results that the increase in age and duration of the disease leads to higher rates of SNHL. The mean age of the patients in our study was significantly higher than this study, and therefore higher rates of SNHL may have been observed. In a study conducted by Kalyoncu et al., in pediatric IBD patients, no difference in hearing loss was found between the patients and the control group (21). Consistent with ours, disease activity and the treatment used were not found to be effective on SNHL in that study. In another study conducted in the pediatric age group, SNHL was detected at high frequencies (10-12.5$16 \mathrm{kHz}$ ), emphasizing that this may be an early indicator of SNHL (22).

Previous studies have shown that medications used for the treatment of IBD (mesalamine, steroid, azathioprine) do not increase the risk of hearing loss $(7,10)$. In the present study, it was shown that mesalazine, steroid, azathioprine and anti TNF's used in UC patients did not affect hearing levels similar to these studies (p:0.69, p:0.82, p:0.50, p:0.42, respectively).
There are several limitations of our study. First of all, since our study is a single-center study, the number of patients and controls is limited. Secondly, our study is a cross-sectional study, and patients were not followed for SNHL for a long time, and it was not determined whether the patients would develop SNHL later. Finally, our study was conducted to include low frequencies (normal hearing frequencies), and high frequencies were not evaluated.

In conclusion; SNHL was detected in $45.2 \%$ (n:14) of UC patients and $13.6 \%$ (n:3) of CD patients in PTA at 0.5-4 $\mathrm{kHz}$. Also, the hearing functions decrease significantly as the age of the patients and the duration of the disease increases. UC patients with a long-term disease or older patients should be evaluated for SNHL. Further prospective randomized controlled studies with larger patient populations will allow evaluating the relationship between UC and SNHL in more detail.

\section{ETHICAL DECLARATIONS}

Ethics Committee Approval: The study was approved by the Bolu Abant İzzet Baysal University Clinical Research Ethics Committee (Date: 07.11.2019, Decision No: 2019/111)

Informed Consent: All patients signed the free and informed consent form.

Referee Evaluation Process: Externally peer-reviewed.

Conflict of Interest Statement: The authors have no conflicts of interest to declare.

Financial Disclosure: The authors declared that this study has received no financial support.

Author Contributions: All of the authors declare that they have all participated in the design, execution, and analysis of the paper, and that they have approved the final version.

Acknowledgments: We would like to thank Abant Izzet Baysal University's otorhinolaryngology department for their contribution in conducting audiometry and other studies.

\section{REFERENCES}

1. Greuter T, Vavricka SR. Extraintestinal manifestations in inflammatory bowel disease-epidemiology, genetics, and pathogenesis. Expert Rev Gastroenterol Hepatol 2019; 13: 307-17.

2. Vavricka SR, Schoepfer A, Scharl M, Lakatos PL, Navarini A, Rogler G. Extraintestinal manifestations of inflammatory Bowel disease. Inflamm Bowel Dis 2015; 21: 1982-92.

3. Garber A, Regueiro M. Extraintestinal manifestations of inflammatory Bowel disease: epidemiology, etiopathogenesis, and management. Curr Gastroenterol Rep 2019; 21: 31.

4. Fousekis FS, Saridi M, Albani E, et al. Ear involvement in inflammatory bowel disease: a review of the literature. J Clin Med Res 2018; 10: 609-14. 
5. Summers RW, Harker L. Ulcerative colitis and sensorineural hearing loss: is there a relationship? J Clin Gastroenterol 1982; 4: 251-2.

6. Lobo DR, Garcia-Berrocal JR, Ramirez-Camacho R. New prospects in the diagnosis and treatment of immune-mediated inner ear disease. World J Methodol 2014; 4: 91-8.

7. Akbayir N, Calis AB, Alkim C, et al. Sensorineural hearing loss in patients with inflammatory bowel disease: a subclinical extraintestinal manifestation. Dig Dis Sci 2005; 50: 1938-45.

8. Karmody CS, Valdez TA, Desai U, Blevins NH. Sensorineural hearing loss in patients with inflammatory bowel disease. Am J Otolaryngol 2009; 30: 166-70.

9. Casella G, Corbetta D, Zolezzi M, et al. Symptomatic sensorineural hearing loss in patients with ulcerative colitis. Tech Coloproctol 2015; 19: 729-31.

10. Wengrower D, Koslowsky B, Peleg U, et al. Hearing Loss in Patients with Inflammatory Bowel Disease. Dig Dis Sci 2016; 61: 2027-32.

11. Mohammed Vashist N, Samaan M, Mosli MH, et al. Endoscopic scoring indices for evaluation of disease activity in ulcerative colitis. Cochrane Database Syst Rev 2018; 1: CD011450.

12. Harvey RF, Bradshaw JM. A simple index of Crohn's-disease activity. Lancet. 1980; 1: 514

13. Clark JG. Uses and abuses of hearing loss classification. ASHA 1981; 23: 493-500.

14. Kuhn M, Heman-Ackah SE, Shaikh JA, Roehm PC. Sudden sensorineural hearing loss: a review of diagnosis, treatment, and prognosis. Trends Amplif 2011; 15: 91-105.

15. McCabe BF. Autoimmune sensorineural hearing loss. Ann Otol Rhinol Laryngol 1979; 88: 585-9.

16. Atturo F, Colangeli R, Bandiera G, Barbara M, Monini S. Can unilateral, progressive or sudden hearing loss be immunemediated in origin? Acta Otolaryngol 2017; 137: 823-8.

17. Gonzalez JLT, Torres JR, Rios YH, Gonzalez MJV, Mendez Saenz MA, Soto-Galindo GA. Extended high-frequency audiometry as early detection of hearing loss in primary Sjogren syndrome. Clin Rheumatol 2017; 36: 2237-41.

18. Di Stadio A, Ralli M. Systemic lupus erythematosus and hearing disorders: literature review and meta-analysis of clinical and temporal bone findings. J Int Med Res 2017; 45: 1470-80.

19. Bouma G, Strober W. The immunological and genetic basis of inflammatory bowel disease. Nat Rev Immunol 2003; 3: 521-33.

20. Goodall AF, Siddiq MA. Current understanding of the pathogenesis of autoimmune inner ear disease: a review. Clin Otolaryngol 2015; 40: 412-9.

21. Kalyoncu D, Urganci N, Calis AB, Ozbal A. Sensorineural hearing loss in pediatric patients with inflammatory bowel disease. Dig Dis Sci 2010; 55: 150-2.

22. Polat E, Cinar Z, Keskindemirci G, et al. Assessment of hearing function in children with inflammatory bowel disease. J Int Adv Otol 2020; 16: 362-6. 